\title{
A case of BV during pregnancy: Best management approach
}

\author{
Your patient is 24 weeks' gestation, and her bacterial vaginosis diagnosis \\ puts her at increased risk for obstetric complications. How do you proceed?
}

\author{
Callie Fox Reeder, MD, and Patrick Duff, MD
}

\section{IN THIS} ARTICLE

\section{BV-infected}

vaginal

secretions

page 39

Oral and

topical

therapies

page 40

\section{Considering}

partner

treatment

page 41
CASE Pregnant woman with abnormal vaginal discharge

A 26-year-old woman (G2P1001) at 24 weeks of gestation requests evaluation for increased frothy, whitish-gray vaginal discharge with a fishy odor. She notes that her underclothes constantly feel damp. The vaginal $\mathrm{pH}$ is 4.5 , and the amine test is positive.

- What is the most likely diagnosis?

- What obstetrical complications may be associated with this condition?

- How should her condition be treated?

\section{Meet our perpetrator}

Bacterial vaginosis (BV) is one of the most common conditions associated with vaginal discharge among women of reproductive age. It is characterized by a polymicrobial alteration of the vaginal microbiome, and most distinctly, a relative absence of vaginal

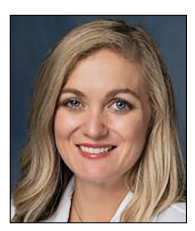

Dr. Reeder is a second-year Fellow, Division of Maternal-Fetal Medicine, Department of Obstetrics and Gynecology, University of Florida College of Medicine, Gainesville.

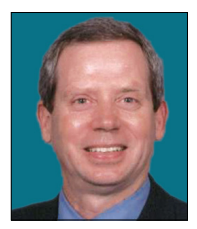

Dr. Duff is Professor of MaternalFetal Medicine, Department of Obstetrics and Gynecology, University of Florida College of Medicine.

The authors report no financial relationships relevant to this article.

doi:10.12788/obgm.0073 lactobacilli. This review discusses the microbiology, epidemiology, specific obstetric and gynecologic complications, clinical manifestations, diagnosis, and treatment of BV.

\section{The role of vaginal flora}

Estrogen has a fundamental role in regulating the normal state of the vagina. In a woman's reproductive years, estrogen increases glycogen in the vaginal epithelial cells, and the increased glycogen concentration promotes colonization by lactobacilli. The lack of estrogen in pre- and postmenopausal women inhibits the growth of the vaginal lactobacilli, leading to a high vaginal $\mathrm{pH}$, which facilitates the growth of bacteria, particularly anaerobes, that can cause BV.

The vaginal microbiome is polymicrobial and has been classified into at least 5 community state types (CSTs). Four CSTs are dominated by lactobacilli. A fifth CST is characterized by the absence of lactobacilli and high concentrations of obligate or facultative anaerobes. ${ }^{1}$ The hydrogen peroxide-producing lactobacilli predominate in normal vaginal flora and make up $70 \%$ to $90 \%$ of the total microbiome. These hydrogen peroxide-producing lactobacilli are associated with reduced vaginal proinflammatory cytokines and a highly acidic vaginal $\mathrm{pH}$. Both factors defend against sexually transmitted infections (STIs). ${ }^{2}$

$\mathrm{BV}$ is a polymicrobial disorder marked by the significant reduction in the number of vaginal lactobacilli (FIGURE 1). A recent 


\section{FIGURE 1 Normal vs bacterial vaginosis vaginal secretions}

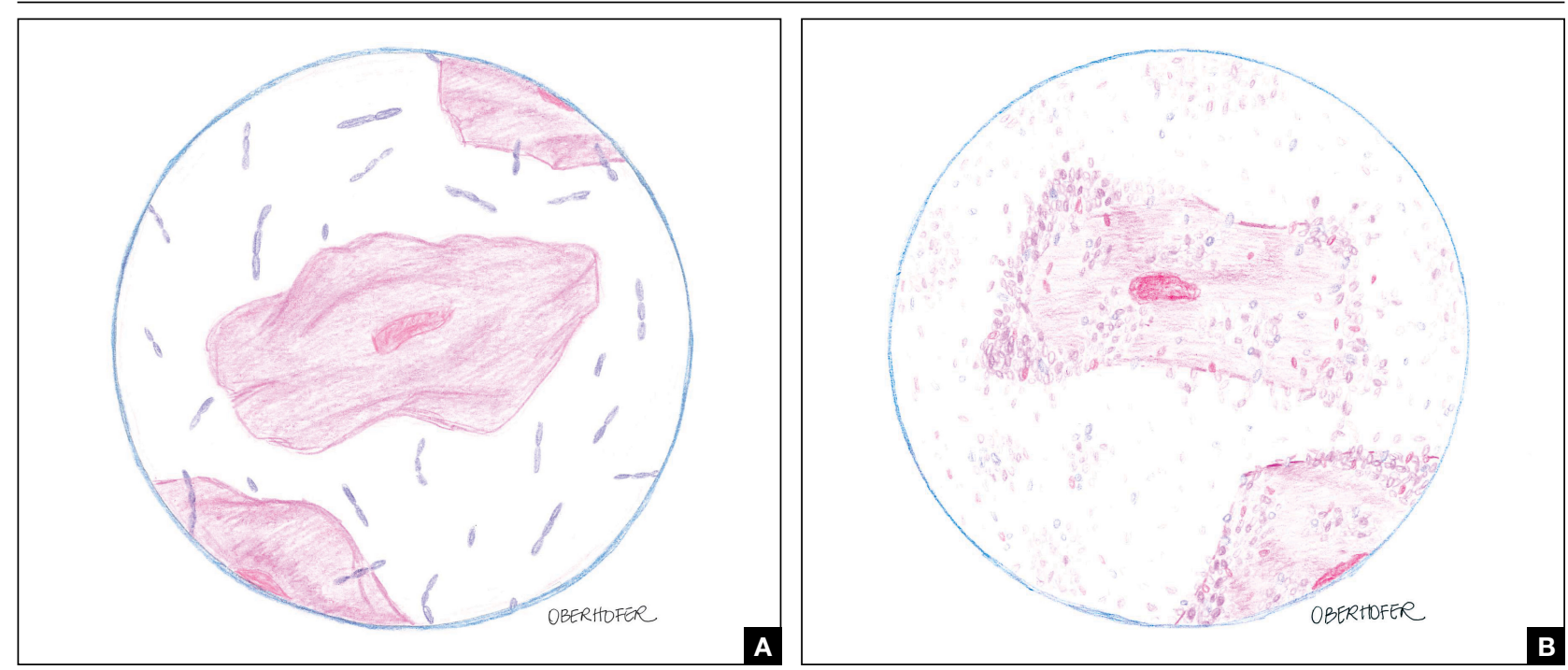

(A) Illustration of Gram stain of normal vaginal secretions. Note the clear borders of the epithelial cells and a "clean" background dominated by lactobacilli. (B) Illustration of Gram stain of vaginal secretions from a patient with bacterial vaginosis. Note the bacteria studding the surface of the epithelial cells (clue cells), the "murky" background, and the absence of lactobacilli.

Illustrations courtesy of Haley Oberhofer.

study showed that BV is associated first with a decrease in Lactobacillus crispatus, followed by increase in Prevotella bivia, Gardnerella vaginalis, Atopobium vaginae, and Megasphaera type $1 .^{3}$ The polymicrobial load is increased by a factor of up to 1,000 , compared with normal vaginal flora. ${ }^{4} \mathrm{BV}$ should be considered a biofilm infection caused by adherence of $G$ vaginalis to the vaginal epithelium. ${ }^{5}$ This biofilm creates a favorable environment for the overgrowth of obligate anaerobic bacteria.

\section{BMI factors into epidemiology}

$\mathrm{BV}$ is the leading cause of vaginal discharge in reproductive-age women. In the United States, the National Health and Nutrition Examination Survey estimated a prevalence of $29 \%$ in the general population and $50 \%$ in Black women aged 14 to 49 years. $^{6}$ In 2013, Kenyon and colleagues performed a systematic review to assess the worldwide epidemiology of BV, and the prevalence varied by country. Within the US population, rates were highest among non-Hispanic, Black women. ${ }^{7}$ Brookheart and colleagues demonstrated that, even after controlling for race, overweight and obese women had a higher frequency of $\mathrm{BV}$ compared with leaner women. In this investigation, the overall prevalence of $\mathrm{BV}$ was $28.1 \%$. When categorized by body mass index (BMI), the prevalence was $21.3 \%$ in lean women, $30.4 \%$ in overweight women, and $34.5 \%$ in obese women $(P<.001)$. The authors also found that Black women had a higher prevalence, independent of BMI, compared with White women. ${ }^{8}$

Complications may occur. BV is notable for having several serious sequelae in both pregnant and nonpregnant women. For obstetric patients, these sequelae include an increased risk of preterm birth; first trimester spontaneous abortion, particularly in the setting of in vitro fertilization; intraamniotic infection; and endometritis. ${ }^{9,10}$ The risk of preterm birth increases by a factor of 2 in infected women; however, most women with BV do not deliver preterm. ${ }^{4}$ The risk of endometritis is increased 6 -fold in women with BV. ${ }^{11}$ Nonpregnant women with BV are at increased risk for pelvic inflammatory disease, postoperative infections, and 


\section{FIGURe 2 Photomicrograph of a saline microscopic preparation showing clue cells}

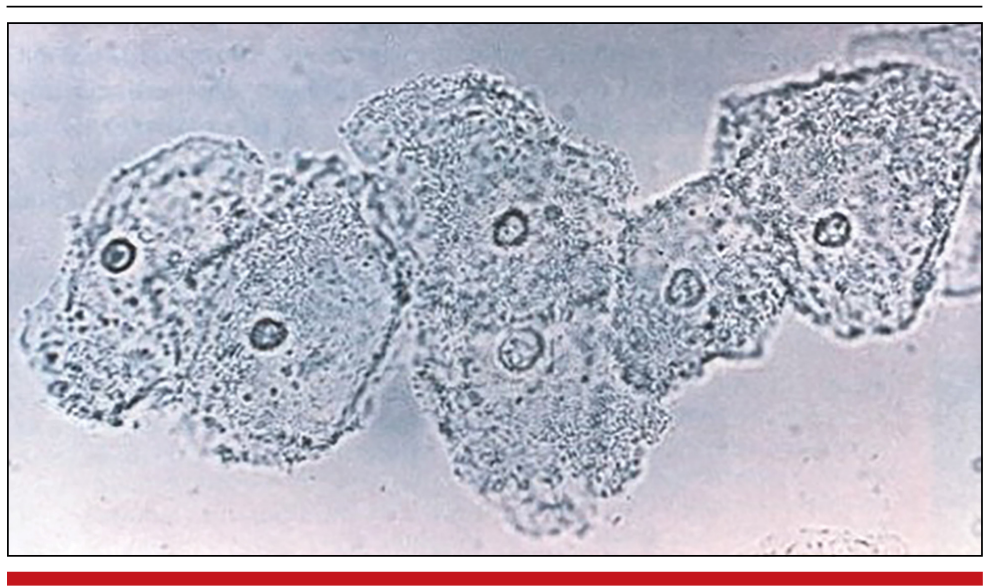

an increased susceptibility to STIs such as chlamydia, gonorrhea, herpes simplex virus, and HIV. ${ }^{12-15}$ The risk for vaginal-cuff cellulitis and abscess after hysterectomy is increased 6 -fold in the setting of BV. ${ }^{16}$

\section{FAST}

TRACK

Up to $50 \%$

of women

treated

for BV

have

disease

recurrence

within

12 months

\section{Clinical manifestations}

$\mathrm{BV}$ is characterized by a milky, homogenous, and malodorous vaginal discharge accompanied by vulvovaginal discomfort and vulvar irritation. Vaginal inflammation typically is absent. The associated odor is fishy, and this odor is accentuated when potassium hydroxide $(\mathrm{KOH})$ is added to the vaginal discharge (amine or "whiff" test) or after the patient has coitus. The distinctive odor is due to the release of organic acids and polyamines that are byproducts of anaerobic bacterial metabolism of putrescine and cadaverine. This release is enhanced by exposure of vaginal secretions to alkaline substances such as $\mathrm{KOH}$ or semen.

Diagnostic tests and criteria. The diagnosis of BV is made using Amsel criteria or Gram stain with Nugent scoring; bacterial culture is not recommended. Amsel criteria include:

- homogenous, thin, white-gray discharge

- $>20 \%$ clue cells on saline microscopy (FIGURE 2)

- a pH >4.5 of vaginal fluid

- positive $\mathrm{KOH}$ whiff test.
For diagnosis, 3 of the 4 Amsel criteria must be present. ${ }^{17}$ Gram stain with Nugent score typically is used for research purposes. Nugent scoring assigns a value to different bacterial morphotypes on Gram stain of vaginal secretions. A score of 7 to 10 is consistent with $\mathrm{BV}^{18}$

\section{Oral and topical treatments}

Treatment is recommended for symptomatic patients. Treatment may reduce the risk of transmission and acquisition of other STIs. The TABLE summarizes Centers for Disease Control and Prevention (CDC) guidelines for BV treatment, ${ }^{19}$ with options including both oral and topical regimens. Oral and topical metronidazole and oral and topical clindamycin are equally effective at eradicating the local source of infection ${ }^{20}$; however, only oral metronidazole and oral clindamycin are effective in preventing the systemic complications of BV. Oral metronidazole has more adverse effects than oral clindamycin-including nausea, vomiting, diarrhea, and a disulfiram-like reaction (characterized by flushing, dizziness, throbbing headache, chest and abdominal discomfort, and a distinct hangover effect in addition to nausea and vomiting). However, oral clindamycin can cause antibiotic-associated colitis and is more expensive than metronidazole.

Currently, there are no single-dose regimens for the treatment of BV readily available in the United States. Secnidazole, a 5-nitroimidazole with a longer half-life than metronidazole, ( 17 vs 8 hours) has been used as therapy in Europe and Asia but is not yet available commercially in the United States. ${ }^{21}$ Hiller and colleagues found that $1 \mathrm{~g}$ and $2 \mathrm{~g}$ secnidazole oral granules were superior to placebo in treating $B .^{22} \mathrm{~A}$ larger randomized trial comparing this regimen to standard treatment is necessary before this therapy is adopted as the standard of care.

Managing recurrent disease, a common problem. Bradshaw and colleagues noted that, although the initial treatment of $\mathrm{BV}$ is effective in approximately $80 \%$ of women, up to $50 \%$ have a recurrence within 12 months. ${ }^{23}$ Data are limited regarding optimal treatment 
TABLE Centers for Disease Control and Prevention's recommended treatments for bacterial vaginosis ${ }^{19}$

\begin{tabular}{l|l:l}
\hline Medication & \multicolumn{1}{c}{ Regimen } & Price \\
\hline Metronidazole tablets & $500 \mathrm{mg}$ orally twice daily for 7 days & $\$ 8.00-\$ 10.00$ \\
\hline Metronidazole $0.75 \%$ vaginal gel & One full applicator $(5 \mathrm{~g})$ intravaginally daily for 5 days & $\$ 36.00-\$ 83.00$ \\
\hline Clindamycin $\%$ vaginal cream & One full applicator $(5 \mathrm{~g})$ intravaginally at bedtime for 7 days & $\$ 198.00-\$ 204.00$ \\
\hline Alternative treatments & & $\$ 14.00-\$ 20.00$ \\
\hline Tinidazole tablets & 2 g orally once daily for 2 days & $\$ 17.00-\$ 22.00$ \\
\hline Tinidazole tablets & $1 \mathrm{~g}$ orally once daily for 5 days & $\$ 9.00-\$ 12.00$ \\
\hline Clindamycin capsule & 300 mg orally twice daily for 7 days & $\$ 181.00-\$ 187.00$ \\
\hline Clindamycin ovules & 100 mg intravaginally once at bedtime for 3 days &
\end{tabular}

for recurrent infections; however, most regimens consist of some form of suppressive therapy. One regimen includes one full applicator of metronidazole vaginal gel $0.75 \%$ twice weekly for 6 months. ${ }^{24} \mathrm{~A}$ second regimen consists of vaginal boric acid capsules $600 \mathrm{mg}$ once daily at bedtime for 21 days. Upon completion of boric acid therapy, metronidazole vaginal gel $0.75 \%$ should be administered twice weekly for 6 months. ${ }^{25} \mathrm{~A}$ third option is oral metronidazole $2 \mathrm{~g}$ and fluconazole $250 \mathrm{mg}$ once every month. ${ }^{26}$ Of note, boric acid can be fatal if consumed orally and is not recommended during pregnancy.

Most recently, a randomized trial evaluated the ability of $L$ crispatus to prevent BV recurrence. After completion of standard treatment therapy with metronidazole, women were randomly assigned to receive vaginally administered $L$ crispatus (152 patients) or placebo (76 patients) for 11 weeks. In the intention-to-treat population, recurrent BV occurred in $30 \%$ of patients in the $L$ crispatus group and $45 \%$ of patients in the placebo group. The use of $L$ crispatus significantly reduced recurrence of $\mathrm{BV}$ by onethird $(P=.01 ; 95 \%$ confidence interval $[\mathrm{CI}]$, 0.44-0.87). ${ }^{27}$ These findings are encouraging; however, confirmatory studies are needed before adopting this as standard of care.

Should sexual partners be treated as well? BV has not traditionally been considered an STI, and the CDC does not currently recommend treatment of partners of women who have BV. However, in women who have sex with women, the rate of BV concordance is high, and in women who have sex with men, coitus can clearly influence disease activity. Therefore, in patients with refractory $\mathrm{BV}$, we recommend treatment of the sexual partner(s) with metronidazole $500 \mathrm{mg}$ orally twice daily for 7 days. For women having sex with men, we also recommend consistent use of condoms, at least until the patient's infection is better controlled. ${ }^{28}$

\section{CASE Resolved}

The patient's clinical findings are indicative of BV. This condition is associated with an increased risk of preterm delivery and intrapartum and postpartum infection. To reduce the risk of these systemic complications, she was treated with oral metronidazole $500 \mathrm{mg}$ twice daily for 7 days. Within 1 week of completing treatment, she noted complete resolution of the malodorous discharge.

\section{References}

1. Smith SB, Ravel J. The vaginal microbiota, host defence and reproductive physiology. J Physiol. 2017;595:451-463.

2. Mitchell C, Fredricks D, Agnew K, et al. Hydrogen peroxide-producing lactobacilli are associated with lower levels of vaginal interleukin- $1 \beta$, independent of bacterial vaginosis. Sex Transm Infect. 2015;42:358-363.

\section{$\overline{\text { FAST }}$ TRACK \\ Although \\ Lactobacillus \\ crispatus has \\ been shown \\ to reduce \\ $B V$ recurrence, \\ confirmatory \\ study is required \\ to adopt \\ the treatment \\ as standard \\ of care}

CONTINUED ON PAGE 42 
3. Munzy CA, Blanchard E, Taylor CM, et al. Identification of key bacteria involved in the induction of incident bacterial vaginosis: a prospective study. J Infect. 2018;218:966-978.

4. Paavonen J, Brunham RC. Bacterial vaginosis and desquamative inflammatory vaginitis. $N$ Engl J Med. 2018; 379:2246-2254

5. Hardy L, Jespers V, Dahchour N, et al. Unravelling the bacterial vaginosis-associated biofilm: a multiplex Gardnerella vaginalis and Atopobium vaginae fluorescence in situ hybridization assay using peptide nucleic acid probes. PloS One. 2015;10:E0136658.

6. Allswoth JE, Peipert JF. Prevalence of bacterial vaginosis: 2001-2004 national health and nutrition examination survey data. Obstet Gynecol. 2007;109:114-120.

7. Kenyon C, Colebunders R, Crucitti T. The global epidemiology of bacterial vaginosis: a systematic review. Am J Obstet Gynecol. 2013;209:505-523.

8. Brookheart RT, Lewis WG, Peipert JF, et al. Association between obesity and bacterial vaginosis as assessed by Nugent score. Am J Obstet Gynecol. 2019;220:476.el-476.e11.

9. Onderdonk $\mathrm{AB}$, Delaney ML, Fichorova RN. The human microbiome during bacterial vaginosis. Clin Microbiol Rev. 2016;29:223-238.

10. Brown RG, Marchesi JR, Lee YS, et al. Vaginal dysbiosis increases risk of preterm fetal membrane rupture, neonatal sepsis and is exacerbated by erythromycin. BMC Med. 2018;16:9.

11. Watts DH, Eschenbach DA, Kenny GE. Early postpartum endometritis: the role of bacteria, genital mycoplasmas, and chlamydia trachomatis. Obstet Gynecol. 1989;73:52-60.

12. Balkus JE, Richardson BA, Rabe LK, et al. Bacterial vaginosis and the risk of Trichomonas vaginalis acquisition among HIV1-negative women. Sex Transm Dis. 2014;41:123-128.

13. Cherpes TL, Meyn LA, Krohn MA, et al. Association between acquisition of herpes simplex virus type 2 in women and bacterial vaginosis. Clin Infect Dis. 2003;37:319-325.

14. Wiesenfeld HC, Hillier SL, Krohn MA, et al. Bacterial vaginosis is a strong predictor of Neisseria gonorrhoeae and Chlamydia trachomatis infection. Clin Infect Dis. 2003;36:663-668.

15. Myer L, Denny L, Telerant R, et al. Bacterial vaginosis and susceptibility to HIV infection in South African women: a nested case-control study. J Infect. 2005;192:1372-1380.
16. Soper DE, Bump RC, Hurt WG. Bacterial vaginosis and trichomoniasis vaginitis are risk factors for cuff cellulitis after abdominal hysterectomy. Am J Obstet Gynecol. 1990;163:1061-1121.

17. Amsel R, Totten PA, Spiegel CA, et al. Nonspecific vaginitis. diagnostic criteria and microbial and epidemiologic associations. Am J Med. 1983;74:14-22.

18. Nugent RP, Krohn MA, Hillier SL. Reliability of diagnosing bacterial vaginosis is improved by a standardized method of gram stain interpretation. J Clin Microbiol. 1991;29:297-301.

19. Bacterial vaginosis. Centers for Disease Control and Prevention website. Updated June 4, 2015. Accessed December 9, 2020. https://www.cdc.gov/std/tg2015/bv.htm.

20. Oduyebo OO, Anorlu RI, Ogunsola FT. The effects of antimicrobial therapy on bacterial vaginosis in non-pregnant women. Cochrane Database Syst Rev. 2009:CD006055.

21. Videau D, Niel G, Siboulet A, et al. Secnidazole. a 5-nitroimidazole derivative with a long half-life. $\mathrm{Br} J$ Vener Dis. 1978;54:77-80.

22. Hillier SL, Nyirjesy P, Waldbaum AS, et al. Secnidazole treatment of bacterial vaginosis: a randomized controlled trial. Obstet Gynecol. 2017;130:379-386.

23. Bradshaw CS, Morton AN, Hocking J, et al. High recurrence rates of bacterial vaginosis over the course of 12 months after oral metronidazole therapy and factors associated with recurrence. J Infect. 2006;193:1478-1486.

24. Sobel JD, Ferris D, Schwebke J, et al. Suppressive antibacterial therapy with $0.75 \%$ metronidazole vaginal gel to prevent recurrent bacterial vaginosis. Am J Obstet Gynecol. 2006;194:1283-1289.

25. Reichman O, Akins R, Sobel JD. Boric acid addition to suppressive antimicrobial therapy for recurrent bacterial vaginosis. Sex Transm Dis. 2009;36:732-734.

26. McClelland RS, Richardson BA, Hassan WM, et al. Improvement of vaginal health for Kenyan women at risk for acquisition of human immunodeficiency virus type 1: results of a randomized trial. J Infect. 2008;197:1361-1368.

27. Cohen CR, Wierzbicki MR, French AL, et al. Randomized trial of lactin-v to prevent recurrence of bacterial vaginosis. $N$ Engl JMed. 2020;382:906-915.

28. Barbieri RL. Effective treatment of recurrent bacterial vaginosis. OBG Manag. 2017;29:7-12. 